\title{
Characteristics of the vaginal microbiome in cross-border female sex workers in China: a case-control study
}

\author{
Xiang Hong ${ }^{1}$, Shenghao Fang ${ }^{2}$, Kaiping Huang ${ }^{1}$, Jiechen Yin ${ }^{1}$, Jianshuang Chen ${ }^{1}$, Yan Xuan ${ }^{1}$, Jing Zhu $^{1}$, Jun Ma $^{1}$ \\ , Pengfei Qin ${ }^{3}$, Danhong Peng ${ }^{3}$, Ning Wang ${ }^{4}$, Bei Wang ${ }^{\text {Corresp. } 1}$ \\ ${ }^{1}$ Key Laboratory of Environmental Medicine and Engineering of Ministry of Education, Department of Epidemiology and Health Statistics, School of Public \\ Health, Southeast University, Nanjing, China \\ 2 Mount Sinai Health System, New York, USA \\ 3 Department of Obstetrics and Gynecology, Zhong Da Hospital, School of Medicine, Southeast University, Nanjing, China \\ 4 National Center for AIDS/STD Control and Prevention, Chinese Center for Disease Control and Prevention, Beijing, China \\ Corresponding Author: Bei Wang \\ Email address: wangbeilxb@163.com
}

Background : The female sex workers (FSWs) are key groups in the transmission of sexual transmitted infections (STI) and vaginal microbiome variations play an important role for it. We aimed to explore the characteristics of vaginal microbiome among FSWs.

Materials and Methods: 24 cross-border female sex workers were randomly selected from a crosssectional survey for female sex workers in southwest China. 37 female non-sex workers were randomly selected from the gynecology clinic and health examination center. Vaginal swabs were collected, bacterial DNA extracted and 16S rRNA genes were sequenced. Differences in the vaginal microbiome between both groups were compared using bioinformatics analysis.

Results: One DNA sample was excluded due to unqualified concentration, therefore 60 samples were sequenced. Female sex workers had significantly different vaginal microbiota $\beta$ diversity, but undifferentiated $\alpha$ diversity when compared with non-sex workers. The average relative abundance of Sneathia, Shigella, Neisseria, Chlamydia, Prevotella, Enterococcus and Ureaplasma among female sex workers was higher than female non-sex workers, and relative abundance of Atopobium in female sex workers was lower than female non-sex workers. The Lactobacillus genus was the major genus in both groups. At the species level, Lactobacllus crispatus, Lactobacllus gasseri and Lactobacllus jensenii, in female sex workers, were lower when compared to female non-sex workers.

Conclusion: There were distinct differences in vaginal bacteria variety between female sex workers and female non-sex workers. Some disease-related genus were also more abundant in female sex workers. Based on these observations, further research is required to identify microbiome communities related to high STI risks and other diseases in these cohorts. 
1 Titile: Characteristics of the vaginal microbiome in cross-border female sex

2 workers in China: a case-control study

3 Running Head: Vaginal Microbiome in Female Sex Workers

4 Xiang Hong $\mathrm{PhD}^{1}$, Shenghao Fang $\mathrm{MD}^{2}$, Kaiping Huang MS ${ }^{1}$, Jiechen Yin $\mathrm{PhD}^{1}$, Jianshuang Chen MS ${ }^{1}$, Yan Xuan

$5 \mathrm{PhD}^{1}$, Jing Zhu $\mathrm{PhD}^{1}$, Jun $\mathrm{Ma}^{1}$, Pengfei Qin $\mathrm{MS}^{3}$, Danhong Peng $\mathrm{MD}^{3}$, Ning Wang $\mathrm{PhD}^{4}$, Bei Wang $\mathrm{PhD}^{1 *}$

7 1. Key Laboratory of Environmental Medicine and Engineering of Ministry of Education, Department of Epidemiology and Health Statistics, School of Public Health, Southeast University, Nanjing, Jiangsu, China;

9 2. Mount Sinai Health System, New York, NY USA;

3. Department of Obstetrics and Gynecology, Zhong Da Hospital, School of Medicine, Southeast University, Nanjing 210009, China.

4. National Center for AIDS/STD Control and Prevention, Chinese Center for Disease Control and Prevention, Beijing City, China

Corresponding author: Professor Bei Wang; postcode: 210009, No.87 Dingjiaqiao Rd, Gulou district, Nanjing city,

Jiangsu province, China. Department of Epidemiology and Statistics, School of Public Health, Southeast University.

Tel: +86-02583792569, E-mail:wangbeilxb@163.com

Conflicts of Interest and Source of Funding: Bei Wang has received funding by National Natural Science 
22 For the remaining authors none were declared.

23

Peer) reviewing PDF | (2019:06:38872:1:1:NEW 20 Oct 2019) 


\section{Abstract}

26 Background: The female sex workers (FSWs) are key groups in the transmission of sexual transmitted infections

27 (STI) and vaginal microbiome variations play an important role for it. We aimed to explore the characteristics of

28 vaginal microbiome among FSWs.

Materials and Methods: 24 cross-border female sex workers were randomly selected from a cross-sectional survey

for female sex workers in southwest China. 37 female non-sex workers were randomly selected from the gynecology analysis.

Results: One DNA sample was excluded due to unqualified concentration, therefore 60 samples were sequenced.

Female sex workers had significantly different vaginal microbiota $\beta$ diversity, but undifferentiated $\alpha$ diversity when compared with non-sex workers. The average relative abundance of Sneathia, Shigella, Neisseria, Chlamydia, Prevotella, Enterococcus and Ureaplasma among female sex workers was higher than female non-sex workers, and relative abundance of Atopobium in female sex workers was lower than female non-sex workers. The Lactobacillus genus was the major genus in both groups. At the species level, Lactobacllus crispatus, Lactobacllus gasseri and Lactobacllus jensenii, in female sex workers, were lower when compared to female non-sex workers.

41 Conclusion: There were distinct differences in vaginal bacteria variety between female sex workers and female nonsex workers. Some disease-related genus were also more abundant in female sex workers. Based on these observations, cohorts. 
45 Keywords: female sex workers; vaginal microbiome; Lactobacillus, female non-sex workers

\section{Introduction}

The current evidence suggests that female sex workers (FSWs) are key factors in the transmission of human immunodeficiency virus (HIV) and other sexually transmitted infections (STIs).(Ferreira-Junior et al. 2018) Every year, STIs affect more than 500 million people worldwide and lead to large financial burdens in hospitals, communities and health systems.(Gottlieb et al. 2014) Though most STIs are curable with timely diagnosis and treatment, others may lead to serious long-term effects, including reproductive complications and death, especially among FSWs who are usually clandestine and do not have access to medical support.(Su et al. 2016) Recent epidemiological investigations have observed unique characteristics in FSWs in the border region of Yunnan province, China, where the vast majority of FSWs come from Vietnam. (Wang et al. 2012; Zhu et al. 2018) It is believed, these cross-border FSWs play key roles in STI transmission, because of frequent travelling and their tendencies to hide work histories.(Zhu et al. 2018) The prevalence of STIs and related complications among the general population in local areas is difficult to control. The cross-border sex industry is a complicated social problem, however it is important to understand and recognize STI or HIV risk factors to public health.

The vaginal microenvironment is a vital factor for STI transmission.(van de Wijgert 2017) In general, Lactobacillus is the predominant bacteria in the vaginal flora. The L. crispatus was always regarded as a beneficial bacterium, which breaks down glycogen to produce lactic acid, hydrogen peroxide and other anti-microbial factors to balance the vaginal microflora.(Ravel et al. 2011) Nevertheless, the role of L.iners in the vaginal microbiome was unclear.(Petrova et al. 2017) The vaginal microbiome was usually a low-diversity, lactobacilli-dominated community, otherwise, it would be considered as vaginal dysbiosis,(van de Wijgert 2017) where the mucosal barrier of vaginal 
was easier to be disrupted. Hence the risk of STI infection is higher in women with vaginal dysbiosis.(Ziklo et al. 2018) Similarly, cervico-vaginal inflammation is likely to increase the concentration of lymphocyte, which were target cells for HIV at mucosal sites. When HIV exposure occurs, the infection rates become increased.(Selhorst et al. 2017) Meanwhile, the increased relative abundance of some opportunistic pathogens in the vagina, such as Gardnerella vaginalis and Mycoplasma, are also associated with bacterial vaginitis (BV),(Onderdonk et al. 2016) endometriosis (Campos et al. 2018), infertility (Tsevat et al. 2017) and adverse pregnancy outcomes. (Murtha \& Edwards 2014)

With the development of next generation sequencing, researchers have focused on vaginal microbiome structure and composition effects on human health,(Chen et al. 2017) and not only single bacterial strains. When getting the abundance imformation of all species, the vaginal microbiota community could be classified as different community state types (CSTs) by some mathematic algorithms. The most common type of classification is five-state model, each of which is characterized by a specific and typical composition and an abundance of taxa: CST I is dominated by L.crispatus, CST II is dominated by L.gasseri, CST III is dominated by L.iners, CST IV is highly diverse and CST V is dominated by L.jensenii.(Lewis et al. 2017) Further research has shown that different CSTs have different effects on human health, and that different Lactobacillus species do not have the same benefits.(Petrova et al. 2017) But evidence showing the unique characteristics of vaginal microbiome in FSWs is still lacking. Notwithstanding this, Wessels et al.,(Wessels et al. 2017) reported that high-risk sexual behavior was associated with vaginal microbiota diversity and a lack of Lactobacillus using the 16S high-throughput sequencing technology among Kenyan. In considering that the vaginal microbiome was diverse with different ethnic backgrounds,(Ravel et al. 2011) the characteristics of vaginal microbiome among Asian FSWs is worthy of study. .

Here, we conducted a case control study to determine differences in the vaginal microbiome between FSWs and female non sexual workers (FNSWs) in a border region of China. We hoped to understand the possible effects of 
commercial sex behaviors on the vaginal microbiome, and provide some new thoughts for FSWs management.

\section{MATERIALS AND METHODS}

\section{Study participants}

Vaginal swabs from FSWs was collected in a FSW cross-sectional study conducted by the Chinese Center for Disease Control (CDC) from June to December 2015 in Hekou County, Yunnan Province, China. Elements of this program have been reported in previous studies (Reilly et al. 2012; Wang et al. 2015; Wang et al. 2012; Zhu et al. 2018). All eligible FSWs were over 16 years old and had reported they had provided sexual services in exchange for money within the past six months. Sexual working conditions, smoking status, pregnancy and vaginitis history were self-reported. Some Vietnamese FSWs who could not speak Chinese were interviewed by a translator. For the collection of vaginal swabs, we excluded FSWs who were sexually active, or who had taken antibiotics in the previous 12 hours, or were menstruating. The specific power and sample size estimation of microbiome studies (Kelly et al. 2015 ) pointed that 20 subjects per group allows more than $90 \%$ power to detect an $\omega^{2}$ of 0.03 . So we selected 24 HIV-negative FSWs by random for sequencing analysis (12 Vietnamese and 12 Chinese women). In considertation that we could not completely distinguish the FSW or NFSW women in the local hosipital, for the high proportion of FSWs there and that they would not willing to tell us the real careers, we recruited 37 FNSWs from the gynecology clinic and health examination center in Zhongda hospital, Nanjing city, which is another southern city of China to reduce the potential misclassification bias. Demographic characteristics were collected along with vaginal swabs. One FSW swab was removed because of too low nucleic acid concentration. The CDC granted Ethical approval to carry out the study (Ethical Application Ref: X120331209), and written informed consent was obtained from all participants in the study. 


\section{Vaginal swabs collection}

All participants were placed in a lithotomy position for swab collection. A gynecologist swabbed for vaginal secretions and then examined the vagina with a speculum. A dry sterile swab was used to scrub secretions at the posterior fornix by rotating three times. The swabs were kept in a dry tube with a unique identification number and immediately placed in a $4^{\circ} \mathrm{C}$ sampling collection box. They were then transferred to a $-80^{\circ} \mathrm{C}$ refrigerator, to await nucleic acid extraction. The samples were transported to the laboratory in drikold.

\section{DNA extraction and 16S rRNA gene sequencing}

Swabs were thawed and placed in $1 \mathrm{ml}$ PBS buffer solution. After 10 min oscillate (Water-bathing Constant Temperature Vibrator, Guohua Machinery Co. Ltd, Jiangsu, China), swabs were removed and the PBS eluates centrifuged for $2 \min$ (12000 rpm, Centrifuge 5424 R, Eppendorf Co. Ltd, Hamburg, Germany). Sediments were DNA extracted using TIANamp Bacteria DNA Kit (Tiangen Biochemical Technology, Beijing, China), following manufacturer's instructions. Purified nucleic acids were eluted in TE buffer and quantified on a Nanodrop 2000 (Thermo Fisher Scientific, Canada). As stated, one DNA sample of unqualified concentration $(<10 \mathrm{ng} / \mathrm{ul}$ with an A260/280 ratio outside 1.6 2.0) was removed.

The hyper-variable V3-V4 region of the 16S rRNA gene was amplified by PCR, using modified 338F and 806R primers with a unique $12 \mathrm{bp}$ barcode, facilitating sequencing on the Illumina HiSeq platform (Illumina, San Diego, CA, USA). Primers were synthesized by Invitrogen (Invitrogen, Carlsbad, CA, USA). PCR reactions, containing 25 $\mu 1$ 2x Premix Taq (Takara Biotechnology, Dalian Co. Ltd., China), $1 \mu$ l each primer (10 mM) and $3 \mu 1$ DNA (20 ng/ $\mu l)$ template in a final volume of $50 \mu \mathrm{l}$, were amplified using the following parameters: Initialization for $5 \mathrm{~min}$ at $94^{\circ} \mathrm{C}$; 30 cycles of $30 \mathrm{~s}$ denaturation at $94^{\circ} \mathrm{C}, 30 \mathrm{~s}$ annealing at $52^{\circ} \mathrm{C}$, and $30 \mathrm{~s}$ extension at $72^{\circ} \mathrm{C}$; followed by a $10 \mathrm{~min}$ elongation at $72^{\circ} \mathrm{C}$. PCR was performed on a BioRad S1000 (Bio-Rad Laboratory, CA, USA). The PCR products 
were sequenced on an IlluminaHiseq2500 platform.

\section{Data processing and statistical analysis}

Quality filtering on the paired-end raw reads was performed under specific filtering conditions to obtain highquality clean reads according to the Trimmomatic (V0.33) quality-controlled process. Paired-end clean reads were merged using FLASH(V1.2.7), according to the relationship of the overlap between the paired-end reads. Sequences with $\geq 97 \%$ similarity were assigned to the same operational taxonomic units (OTUs) using the Qiime software (version 1.8.0). For each representative sequence, the silva database (https://www.arb-silva.de/) was used to annotate taxonomic information. OTU abundance information were normalized using a standard of sequence number corresponding to the sample with the least sequences. All bioinformatics analyses were completed on the Biomarker biocloud platform (www.biocloud.org).

Mothur software (version v.1.30) assessed alpha diversity, including ACE, Chao1, and the Simpson and Shannon index (Schloss et al. 2009). Principle Coordinate Analyses (PCoAs) based on Binary_jaccard dissimilarity, permutational multivariate analysis of variance (PERMANOVA) and cluster heatmap were generated to statistically compare microbial populations ( $\beta$ diversity) between groups. Euclidean distances between different samples or genera were calculated, and the z-score transformation for reletive abundance of specific genus was generated to create the cluster heatmap. To illustrate relationships between some environmental factors and microbiota communities, a redundancy analysis (RDA) was performed. Linear Discriminant Analysis (LDA) Effect Size (Lefse), Random Forest analysis and multivariate Kruskal-Wallis test (P was adjusted by the BH-FDR method) were performed to compare the relative abundance of specific genus between groups. These statistical methods were performed on the Biomarker biocloud platform using related $\mathrm{R}$ packages. To identify specific Lactobacillus species, representative sequences were queried against NCBI's 16S rRNA gene database using BLAST. The highest scoring species $(>97 \%$ identity and 
149

150

151

152

153

154

155

156

157

158

159

160

161

162

163

164

165

166

167

168

coverage) was selected as the putative identity of that OTU. Community state types of vaginal microbiome were defined using Jensen-Shannon divergence and Ward linkage hierarchical clustering. (Gajer et al. 2012) Scatter diagrams were generated on GraphPad Prism (GraphPad Software Inc., La Jolla, CA).

Continuous variables (age, the number of pregnancies and $\alpha$ diversity indices) were statistically compared across groups using the Mann-Whitney Test, as these data were not normally distributed. Analysis of covariance (ANCOVA) was performed to adjust potential factors. Categorical variables (smoking status, vaginitis history) were presented as frequencies (percentages) and compared between groups by Fisher's Exact Test or Chi-square Test. All analyses were performed on SAS software (version 9.2). For all tests, a two-sided P value less than or equal to 0.05 was deemed statistically significant.

\section{RESULTS}

\section{Participants}

Vaginal swabs were available from 60 women (23 FSWs and 37 FNSWs). Smoking rates between groups was not statistically different. However, the FSWs were younger than the FNSWs (Median 25 vs 29; $\mathrm{P}=0.001$ ), and had a greater number of pregnancies $(\mathrm{P}=0.012)$. The proportion of FSWs with a history of vaginitis was higher than FNSWs ( $\mathrm{P}=0.034)$. (Table 1) By using RDA (Fig 1a) and PERMANOVA analysis (Supplement table 1), although the age and pregnancy history were associated with microbiota communities, they could just explain less than $10 \%$ variance of microbiota community $\left(\mathrm{R}^{2}<0.10\right)$. The FNSWs were all Chinese, but FSWs included Vietnamese and Chinese, the PERMANOVA analysis showed that the vaginal microbiome of FSWs with different nationality was of no statistical significance $\left(R^{2}=0.062, P=0.153\right)$. 
FSWs have significantly different vaginal microbiota $\beta$ diversity, but undifferentiated $\alpha$ diversity when

compared with FNSWs.

Although the ACE, Chaol and Shannon indices for FSWs were higher than FNSWs, the differences were not

statistically significant $(\mathrm{P}>0.05)$. After adjusting for age, the number of pregnancies and vaginitis history, the

differences of ACE, Chao1 and Shannon indices between FSWs and FNSWs were not statistical significant. ( $\mathrm{P}>0.05$;

Supplement table 2). Binary jaccard dissimilarity PCoA analysis showed that microbiota communities in FSWs were

visibly different from FNSWs (Fig 1b). The $\mathrm{R}^{2}$ was 0.321 based on the PERMANOVA Test (Supplement table 1).

The cluster heatmap showed FNSWs were mostly clustered in right and FSWs were in left (Fig 1c), which suggested

that the vaginal microbiome structures of FNSWs and FSWs could be distinguished to some extent. The bacteria

genera list in the vertical axis were the commom genera of which relative abundances were more than $0.1 \%$.

\section{Specific genus comparation between FSWs and FNSWs}

Lefse analysis (Fig 1d) showed differences in microbiota orders, families, genera and species between the two groups. However only five genera reached the threshold LDA score of more than 3.5: FSWs with a higher abundance of Chlamydia, Shigella, Neisseria and Sneathia and FNSWs with a higher abundance of Atopobium (Fig 2a). The MeanDecreaseGini index from the random forest model (Fig 2b) illustrated that Atopobium, Neisseria and Enterococcus were the key genera in distinguishing FSWs' and FNSWs' vaginal microbiota communities. To compare genus differences between these two groups, a multivariate Kruskal-Wallis test was performed (Fig 2c). By FDR adjusting, the results were almost consistent with the results from the Lefse analysis. The average relative abundances of Sneathia, Shigella, Neisseria, Chlamydia, Prevotella, Enterococcus and Ureaplasma in FSWs was higher than those in FNSWs. The Atopobium abundance in FSWs was lower than FNSWs $(\mathrm{P}<0.05)$. The scatter diagrams showed 
191

192

193

194

195

196

197

198

199

200

201

202

203

204

205

206

207

208

that a FSW sample was abnormally with high abundance of Escherichia_Shigella(Fig 3c). No Neisseria was detected

in FNSW samples but was positive in 21(91.3\%) FSWs, and Chlamydia was detected positive in 18(78.3\%) FSWs

but only one in FNSW samples. (Fig 3d, 3e)

\section{The relative abundance of Lactobacillus spp among FSWs and FNSWs}

The Lactobacillus genus was the dominant species in most vaginal microbiota communities (37/60); however

the average relative abundance between FSWs and FNSWs was not statistically significant $(\mathrm{P}=0.373)$ (Fig 4a). With

regards to specific Lactobacillus strains, the relative abundance of L.crispatus, L.gasseri and L.jensenii in FSWs was

lower than for FNSWs, and L.iners abundance was indistinguishable between two groups (Fig $\mathbf{4 b}-\mathbf{f})$. According to

the specific dominant genus, the vaginal microbiota communities were divided into five types: CSTI (L.crispatus),

CSTII (L.gasseri), CSTIII (L.iners), CSTIV (highly diverse dominant) and CSTV (L.jensenii). No CST V microbiota

were observed in our 60 samples. CST distributions were not statistically different between FSWs and FNSWs

$(\mathrm{P}>0.05)$ (Supplemental table 3).

Influencing factors on FSW vaginal microbiota communities

Within the FSW group, RDA analysis revealed that correlations between vaginal microbiota communities and the average number of clients per week, drinking habits and duration of sex working (Supplement figure 1a) were relatively obvious, but with no statistical significance through PCoA and PERMANOVA analyses. (P>0.05)

\section{(Supplement figure 1b-d)}

\section{Discussion}

Peer) reviewing PDF | (2019:06:38872:1:1:NEW 20 Oct 2019) 
southwest China. We found distinct differences in vaginal bacterial structures, but not diversities between FSWs and

212 FNSWs. The average relative abundances of Sneathia, Shigella, Neisseria, Chlamydia, Prevotella, Enterococcus and

Ureaplasma among FSWs was higher than for FNSWs. Lactobacillus was the major genus in both FSWs and FNSWs;

no differences were observed between them for both groups. However this was inconsistent with the findings by

Wessels et al. (Wessels et al. 2017), the most likely reasons were the race, geography, and hygiene practices. But at a

species level, L.crispatus, L.gasseri and L.jensenii in the FSW group were lower in abundance than the FNSW group.

This study filled the gap of characteristics of the vaginal microbiome in cross-border FSWs in China..

The public health implications of this study are in two aspects as follows. Firstly, the data about FSWs, especially

in cross-border areas, was valuable because the cross-border sexual work industry in China is illegal. In order to ensure

the quality of investigation, this program was conducted by Chinese Center for Disease Control whose remit is to

control HIV transmission (Wang et al. 2012), and the samples were collected by standardized process. What we found

could provide data for further research in this special group. Secondly, most FSWs here were very young or even

juveniles, acquiring STIs or BV or vaginal dysbiosis will affect them going forward. Because most FSWs will go

home to get married, we have reasons to believe that the negative effects of vaginal dysbiosis may impact their future

family, such as STI transmission to husbands. The risk of infertility (Campisciano et al. 2017) or adverse pregnancy

may be higher because of the disorganized vaginal microbiomes.

pathogens (Cabecinha et al. 2017), such as Treponema pallidum, Trichomonad, Neisseria and Chlamydia. Our study 
232

risk of pathogen infection.(Ziklo et al. 2018) We could not illuminate whether the different diversity of vaginal microbiome between FSWs and FNSWs was from the infection of Neisseria / Chlamydia. But in consideration of the high prevalence of STIs, if we made a strict standard to exclude them, the eligible FSWs would be limited and some unpredictable selection biases might exist. Moreover, the changes of relative abundance of Neisseria and Chlamydia were a typical performance of microbiota changes, and the complex correlations between the vaginal species were not explained clearly, so exploring the whole vaginal microbiota communities which were not specially selected were valuable. Meanwhile, the increased relative abundance of Sneathia, Streptococcus and Shigella among FSWs were observed. Sneathia is a Gram-negative, rod-shaped, non-spore-forming and non-motile bacteria; it has the potential to impact on bacterial vaginosis and cause preterm births (Harwich et al. 2012). Streptococcus, especially the Group B Streptococcus (GBS), is always detected in the female genital tract, and is an important pathogen causing adverse pregnancy outcomes and neonatal infections. Rosen et al. (Rosen et al. 2017) observed that vaginal microbiota CST and $\alpha$-diversity are not related to GBS status, however, specific microbial taxa are associated with the colonization of this important human pathogen. Shigella is a pathogenic enterococci typically located in the intestinal tract. When it invades the vagina, the pathogen causes severe vaginitis symptoms, including bloody vaginal discharge (Murphy \& Nelson 1979). It should be noted that anal sex behaviors were common among FSWs (Kelly-Hanku et al. 2014), which may explain the increasing of entero-associated bacterial infections. Atopobium could be detected in healthy women, but its role was controversial. It is commonly associated with bacterial vaginosis and an increasing risk of adverse pregnancy outcomes (Mendes-Soares et al. 2015). But genetic differences across Atopobium species suggests that single species could be associated with both health and disease (Mendes-Soares et al. 2015). In the FNSW group, the relative abundance of the Atopobium genus was higher, but the specific species was unknown. In the future, metagenomic sequencing applications could identify the Atopobium species. 
Although there were no statistical significance of relative abundance of Lactobacillus genus among FSWs and

254

255

256

257

258

259

260

261

262

263

264

265

266

267

268

269

270

271

272

273

FNSWs for our small sample size, the specific species of Lactobacillus were different between groups. L. crispatus

in FNSWs was higher than in FSWs, which plays a crucial role in protecting the genitourinary tract against pathological conditions. Antonietta et al. (Rizzo et al. 2015) found that L.crispatus reduced IL-6, IL-8 and TNF- $\alpha$ production, but specifically enhanced IL-10 anti-inflammatory cytokines in C. trachomatis-infected HeLa and J774 cells, suggesting specific $L$. crispatus protective mechanisms at the vagina. But the effect of $L$.iners on vaginal health was unclear. Biochemical and functional assays have suggested that $L$. iners contain features of probiotic lactobacilli as well as vaginal pathogens, which may be based on genome clonal variants (Petrova et al. 2017). Some studies have shown that $L$. iners dominated vaginal microbiome community appear to be less stable or more in transition than other community types and are associated with vaginal dysbiosis (Verstraelen et al. 2009). Our results revealed that the average relative abundance of $L$. iners among FSWs was higher than for FNSWs, though the differences were not statistically significant. Similarly, the abundance of L. gasseri among FSWs was lower than for FNSWs, which was consistent with Backer's study (De Backer et al. 2007). The negative association between L. iners and L. gasseri further suggested that bacteria in vaginal communities are interrelated.

At present, the CST is the recognized classification method (Gajer et al. 2012), each type is characterized by a specific and typical composition and abundance of taxa. Most vaginal communities and corresponding CSTs are dominated by one or several Lactobacillus species,(Ravel et al. 2011) referred to as CSTI (L.crispatus), CSTII (L.gasseri), CSTIII (L.iners), CSTIV (high diversity dominant) and CSTV (L.jensenii). The associations between different CSTs and health had been studied in other populations (Campisciano et al. 2017; Chen et al. 2017; Rosen et al. 2017; Wessels et al. 2017), but results have been inconsistent. In our analysis, we used this classification method to compare differences in CST composition between FSWs and FNSWs, however no meaningful differences were 
274

observed. Some researchers have suggested that vaginal microbiome stability is usually not expressed in terms of changes in taxa composition, but rather in terms of CST consistency (Gajer et al. 2012). Therefore, we should conduct future studies on associations between CST changes and health status, not just the current CST situation in one time.

The present study was limited due to its cross-sectional elements. The vaginal microbiome is ecologically dynamic, (Greenbaum et al. 2018) therefore going forward, the best approach is to explore the different structures of the vaginal microbiome, between these two population, over at least one menstruation cycle.(Uchihashi et al. 2015) Meanwhile, our cross-sectional study could not explain the causality between STIs and vaginal microbiome changes, and whether commercial sex behaviors led STIs first or impact vaginal microbiome first. Given the number of factors that impact the vaginal microbiome, our data only scratches the surface. For instance, lubricant use and menstruation data among FSWs, and FNSW sexual behaviors were not collected. Equally, information bias was inevitable because of our sensitive questions. In addition, the small sample size may have reduced adequate cohort representation, although our samples were randomly selected, and the inclusion criteria were strict. What's more, Because of the specific characteristics of FSWs, our FSW participants were younger and had higher pregnancy and vaginitis histories, which affected comparability within the groups. Meanwhile, our control group just included the Chinese FNSWs, wihout Vietnamese, which was one of the source of heterogeneity beween FSWs and FNSWs. Our results should be cautiously extrapolated to FSWs in other jurisdictions, because cross-border FSWs in China have different lives and sexual features.

\section{Conclusions}

Our results have indicated distinct differences in vaginal bacterial structures between FSWs and FNSWs; some disease-related genera, such as Sneathia, Shigella, Neisseria and Chlamydia, were more abundant in FSWs. Because of the important health effects of the vaginal microbiome and the high transience of FSWs, further research to identify 
295

296

297

298

299

300

301

302

303

304

305

306

307

308

309

310

311

312

313

314

315

316

317

318

319

320

321

322

323

324

325

326

327

328

specific types of microbiome structures related to FSWs or STI risks is warranted..

Acknowledgements: We thank the staff at CDC in Hekou County for their work on data collection and International

Science Editing for English language editing.

Data sharing statement: The original sequencing data were available from Figshare (www.figshare.com).

\section{Reference}

Cabecinha M, Mercer CH, Gravningen K, Aicken C, Jones KG, Tanton C, Wellings K, Sonnenberg P, and Field N. 2017. Finding sexual partners online: prevalence and associations with sexual behaviour, STI diagnoses and other sexual health outcomes in the British population. Sexually Transmitted Infections 93:572-582. 10.1136/sextrans-2016-052994

Campisciano G, Florian F, D'Eustacchio A, Stankovic D, Ricci G, De Seta F, and Comar M. 2017. Subclinical alteration of the cervical-vaginal microbiome in women with idiopathic infertility. J Cell Physiol 232:16811688. $10.1002 /$ jcp. 25806

Campos GB, Marques LM, Rezende IS, Barbosa MS, Abrao MS, and Timenetsky J. 2018. Mycoplasma genitalium can modulate the local immune response in patients with endometriosis. Fertil Steril 109:549-560.e544. 10.1016/j.fertnstert.2017.11.009

Chen C, Song XL, Wei WX, Zhong HZ, Dai JJ, Lan Z, Li F, Yu XL, Feng Q, Wang ZR, Xie HL, Chen XM, Zeng CW, Wen B, Zeng LP, Du H, Tang HR, Xu CL, Xia Y, Xia HH, Yang HM, Wang J, Wang J, Madsen L, Brix S, Kristiansen K, Xu X, Li JH, Wu RF, and Jia HJ. 2017. The microbiota continuum along the female reproductive tract and its relation to uterine-related diseases. Nature Communications 8. ARTN 875

10.1038/s41467-017-00901-0

De Backer E, Verhelst R, Verstraelen H, Alqumber MA, Burton JP, Tagg JR, Temmerman M, and Vaneechoutte M. 2007. Quantitative determination by real-time PCR of four vaginal Lactobacillus species, Gardnerella vaginalis and Atopobium vaginae indicates an inverse relationship between $\mathrm{L}$. gasseri and L. iners. $B M C$ Microbiol 7:115. 10.1186/1471-2180-7-115

Dunlop AL, Mulle JG, Ferranti EP, Edwards S, Dunn AB, and Corwin EJ. 2015. Maternal Microbiome and Pregnancy Outcomes That Impact Infant Health: A Review. Adv Neonatal Care 15:377-385. 10.1097/anc.0000000000000218

Ferreira-Junior ODC, Guimaraes MDC, Damacena GN, de Almeida WDS, de Souza-Junior PRB, and Szwarcwald CL. 2018. Prevalence estimates of HIV, syphilis, hepatitis B and C among female sex workers (FSW) in Brazil, 2016. Medicine (Baltimore) 97:S3-s8. 10.1097/md.0000000000009218

Gajer P, Brotman RM, Bai G, Sakamoto J, Schutte UM, Zhong X, Koenig SS, Fu L, Ma ZS, Zhou X, Abdo Z, Forney LJ, and Ravel J. 2012. Temporal dynamics of the human vaginal microbiota. Sci Transl Med 4:132ra152. 
10.1126/scitranslmed.3003605

Gottlieb SL, Low N, Newman LM, Bolan G, Kamb M, and Broutet N. 2014. Toward global prevention of sexually transmitted infections (STIs): the need for STI vaccines. Vaccine 32:1527-1535. 10.1016/j.vaccine.2013.07.087

Greenbaum S, Greenbaum G, Moran-Gilad J, and Weintruab AY. 2018. Ecological dynamics of the vaginal microbiome in relation to health and disease. Am J Obstet Gynecol:pii: 0002-9378(0018)32114-32118. [Epub ahead of print]. 10.1016/j.ajog.2018.11.1089

Harwich MD, Jr., Serrano MG, Fettweis JM, Alves JM, Reimers MA, Buck GA, and Jefferson KK. 2012. Genomic sequence analysis and characterization of Sneathia amnii sp. nov. BMC Genomics 13 Suppl 8:S4. 10.1186/1471-2164-13-s8-s4

Kelly-Hanku A, Rawstorne P, Kupul M, Worth H, Shih P, and Man WY. 2014. Anal sex, vaginal sex and HIV risk among female sex workers in Papua New Guinea. AIDS Behav 18:573-582. 10.1007/s10461-013-0624-8

Kelly BJ, Gross R, Bittinger K, Sherrill-Mix S, Lewis JD, Collman RG, Bushman FD, and Li H. 2015. Power and sample-size estimation for microbiome studies using pairwise distances and PERMANOVA. Bioinformatics 31:2461-2468. 10.1093/bioinformatics/btv183

Lewis FM, Bernstein KT, and Aral SO. 2017. Vaginal Microbiome and Its Relationship to Behavior, Sexual Health, and Sexually Transmitted Diseases. Obstet Gynecol 129:643-654. 10.1097/aog.0000000000001932

Mendes-Soares H, Krishnan V, Settles ML, Ravel J, Brown CJ, and Forney LJ. 2015. Fine-scale analysis of 16S rRNA sequences reveals a high level of taxonomic diversity among vaginal Atopobium spp. Pathog Dis 73. $10.1093 / \mathrm{femspd} / \mathrm{ftv} 020$

Murphy TV, and Nelson JD. 1979. Shigella vaginitis: report of 38 patients and review of the literature. Pediatrics 63:511-516.

Murtha AP, and Edwards JM. 2014. The role of Mycoplasma and Ureaplasma in adverse pregnancy outcomes. Obstet Gynecol Clin North Am 41:615-627. 10.1016/j.ogc.2014.08.010

Onderdonk AB, Delaney ML, and Fichorova RN. 2016. The Human Microbiome during Bacterial Vaginosis. Clin Microbiol Rev 29:223-238. 10.1128/cmr.00075-15

Petrova MI, Reid G, Vaneechoutte M, and Lebeer S. 2017. Lactobacillus iners: Friend or Foe? Trends in Microbiology 25:182-191. 10.1016/j.tim.2016.11.007

Ravel J, Gajer P, Abdo Z, Schneider GM, Koenig SSK, McCulle SL, Karlebach S, Gorle R, Russell J, Tacket CO, Brotman RM, Davis CC, Ault K, Peralta L, and Forney LJ. 2011. Vaginal microbiome of reproductive-age women. Proc Natl Acad Sci U S A 108:4680-4687. 10.1073/pnas.1002611107

Reilly KH, Wang J, Zhu Z, Li S, Yang T, Ding G, Qian HZ, Kissinger P, and Wang N. 2012. HIV and associated risk factors among male clients of female sex workers in a Chinese border region. Sex Transm Dis 39:750-755. 10.1097/OLQ.0b013e31825f7af7

Rizzo A, Fiorentino M, Buommino E, Donnarumma G, Losacco A, and Bevilacqua N. 2015. Lactobacillus crispatus mediates anti-inflammatory cytokine interleukin-10 induction in response to Chlamydia trachomatis infection in vitro. Int J Med Microbiol 305:815-827. 10.1016/j.ijmm.2015.07.005

Rosen GH, Randis TM, Desai PV, Sapra KJ, Ma B, Gajer P, Humphrys MS, Ravel J, Gelber SE, and Ratner AJ. 2017. Group B Streptococcus and the Vaginal Microbiota. J Infect Dis 216:744-751. 10.1093/infdis/jix395

Schloss PD, Westcott SL, Ryabin T, Hall JR, Hartmann M, Hollister EB, Lesniewski RA, Oakley BB, Parks DH, Robinson CJ, Sahl JW, Stres B, Thallinger GG, Van Horn DJ, and Weber CF. 2009. Introducing mothur: 
370

371

372

373

374

375

376

377

378

379

380

381

382

383

384

385

386

387

388

389

390

391

392

393

394

395

396

397

398

399

400

401

402

403

404

405

406

407

408

409 open-source, platform-independent, community-supported software for describing and comparing microbial communities. Appl Environ Microbiol 75:7537-7541. 10.1128/AEM.01541-09

Selhorst P, Masson L, Ismail SD, Samsunder N, Garrett N, Mansoor LE, Karim QA, Karim SSA, Passmore JAS, and Williamson C. 2017. Cervicovaginal Inflammation Facilitates Acquisition of Less Infectious HIV Variants. Clinical Infectious Diseases 64:79-82. 10.1093/cid/ciw663

Stout MJ, Zhou Y, Wylie KM, Tarr PI, Macones GA, and Tuuli MG. 2017. Early pregnancy vaginal microbiome trends and preterm birth. Am J Obstet Gynecol 217:356.e351-356.e318. 10.1016/j.ajog.2017.05.030

Su S, Chow EP, Muessig KE, Yuan L, Tucker JD, Zhang X, Ren J, Fairley CK, Jing J, and Zhang L. 2016. Sustained high prevalence of viral hepatitis and sexually transmissible infections among female sex workers in China: a systematic review and meta-analysis. BMC Infect Dis 16:2. 10.1186/s12879-015-1322-0

Tsevat DG, Wiesenfeld HC, Parks C, and Peipert JF. 2017. Sexually transmitted diseases and infertility. Am J Obstet Gynecol 216:1-9. 10.1016/j.ajog.2016.08.008

Uchihashi M, Bergin IL, Bassis CM, Hashway SA, Chai D, and Bell JD. 2015. Influence of age, reproductive cycling status, and menstruation on the vaginal microbiome in baboons (Papio anubis). Am J Primatol 77:563-578. 10.1002/ajp. 22378

van de Wijgert J. 2017. The vaginal microbiome and sexually transmitted infections are interlinked: Consequences for treatment and prevention. PLoS Med 14:e1002478. 10.1371/journal.pmed.1002478

Verstraelen H, Verhelst R, Claeys G, De Backer E, Temmerman M, and Vaneechoutte M. 2009. Longitudinal analysis of the vaginal microflora in pregnancy suggests that L. crispatus promotes the stability of the normal vaginal microflora and that L. gasseri and/or L. iners are more conducive to the occurrence of abnormal vaginal microflora. BMC Microbiol 9:116. 10.1186/1471-2180-9-116

Wang J, Ding G, Zhu Z, Zhou C, and Wang N. 2015. Analysis of HIV Correlated Factors in Chinese and Vietnamese Female Sex Workers in Hekou, Yunnan Province, a Chinese Border Region. PLoS One 10:e0129430. 10.1371/journal.pone.0129430

Wang JJ, Zhu ZB, Yang X, Wu J, Wang HB, Feng L, Ding GW, Norris JL, and Wang N. 2012. Herpes simplex virus type 2 risks in female sex workers in the China-Vietnam border county of Hekou. Biomed Environ Sci 25:706-710. 10.3967/0895-3988.2012.06.013

Wessels JM, Lajoie J, Vitali D, Omollo K, Kimani J, Oyugi J, Cheruiyot J, Kimani M, Mungai JN, Akolo M, Stearns JC, Surette MG, Fowke KR, and Kaushic C. 2017. Association of high-risk sexual behaviour with diversity of the vaginal microbiota and abundance of Lactobacillus. PLoS One 12:e187612. 10.1371/journal.pone.0187612

Zhu J, Yuan R, Hu D, Zhu Z, Wang N, and Wang B. 2018. HIV prevalence and correlated factors of female sex workers and male clients in a border region of Yunnan Province, China. Int J STD AIDS 29:424-434. 10.1177/0956462417730258

Ziklo N, Vidgen ME, Taing K, Huston WM, and Timms P. 2018. Dysbiosis of the Vaginal Microbiota and Higher Vaginal Kynurenine/Tryptophan Ratio Reveals an Association with Chlamydia trachomatis Genital Infections. Frontiers in Cellular and Infection Microbiology 8. ARTN 1

$10.3389 /$ fcimb. 2018.00001 
$410 \quad$ legends

411 Fig 1. Microbiota community diversity from female sex workers and non-sex workers. a) RDA analysis for

412 correlations between microbiota diversity and age, pregnancy histrory and vaginitis histrory of participants; b)

413 Binary_jaccard dissimilarity PCoA analysis for FSWs and FNSWs group; c) Cluster heatmap for different samples

414 and genus; the gradation of color depends on the z-score of the relative abundance for specific genus. d) Lefse analysis

415 for the microbiota between FSW and FNSW groups, threshold LDA score=2; FSW, female sex worker; FNSW, female

416 non-sex worker.

417

418

419

Fig 2. The specific genus between female sex workers and non-sex workers. a) Lefse analysis for genus levels

between FSWs and FNSWs, LDA score > 3.5; b) MeanDecreaseGini index from random forest model; c) The average

relative abundance of specific genera between FSWs and FNSWs; the asterisk relates to the P value of multivariate

Kruskal-Wallis test was less than 0.05 by FDR adjustment. FSW, female sex worker; FNSW, non-sex worker.

Fig 3. The scatter diagrams of specific genera between female sex workers and non-sex workers. The $P$ value

was from multivariate Kruskal-Wallis test and adjusted by FDR. FSW, female sex worker; FNSW, female non-sex worker.

Fig 4. The relative abundance of Lactobacillus between female sex workers and non-sex workers. The $P$ value 
431 Supplement Figure. Exploration for influencing factors associated with FSW vaginal microbiota communities. 


\section{Table 1 (on next page)}

Characteristics of female sex workers and female non-sex workers 
1 Table 1. Characteristics of female sex workers and female non-sex workers

\begin{tabular}{|c|c|c|c|c|}
\hline & $\begin{array}{l}\text { Female Sex Workers } \\
\qquad \mathrm{N}=23\end{array}$ & $\begin{array}{c}\text { Female Non-Sex } \\
\text { Workers } \\
\mathrm{N}=37\end{array}$ & $\mathrm{Z} / \chi^{2}$ & $\mathrm{P}$ \\
\hline Age(Median, Range), Year & $25(18-41)$ & $29(20-40)$ & -3.368 & 0.001 \\
\hline Smoke (Yes), n(\%) & $5(21.7)$ & $8(21.6)$ & & $0.999 *$ \\
\hline Pregnancy history(Median, Range) & $1(0-5)$ & $1(0-3)$ & -2.513 & 0.012 \\
\hline Unknow & 0 & 3 & & \\
\hline Vaginitis history (Yes), n(\%) & $11(47.8)$ & $8(21.6)$ & 4.501 & 0.034 \\
\hline \multicolumn{5}{|l|}{ Nationality } \\
\hline Chinese & 12 & 37 & -- & -- \\
\hline Vietnamese & 11 & 0 & & \\
\hline
\end{tabular}

$2 *$ Fisher's Exact Test

3 


\section{Figure 1}

Microbiota community diversity from female sex workers and non-sex workers.

a) RDA analysis for correlations between microbiota diversity and age, pregnancy histrory and vaginitis histrory of participants; b) Binary jaccard dissimilarity PCoA analysis for FSWs and FNSWs group; c) Cluster heatmap for different samples and genus; d) Lefse analysis for the microbiota between FSW and FNSW groups, threshold LDA score=2; FSW, female sex worker; FNSW, female non-sex worker. 


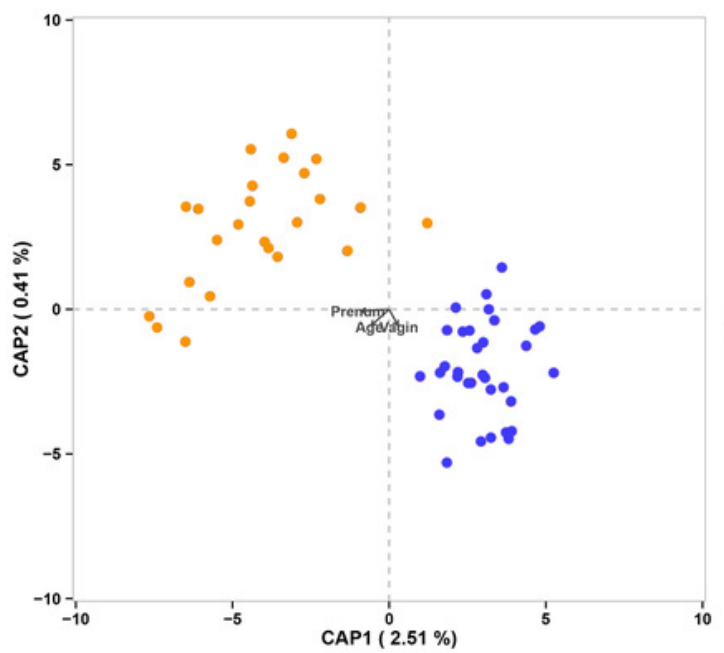

a )

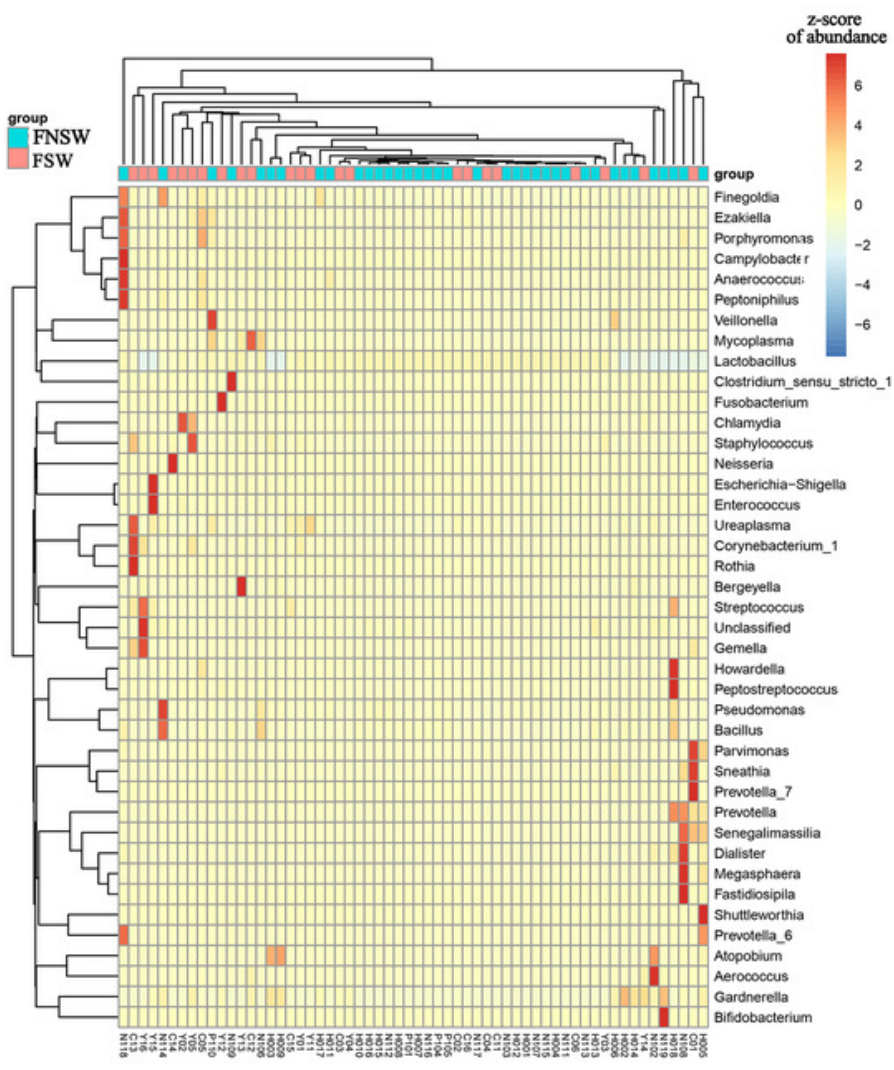

c)
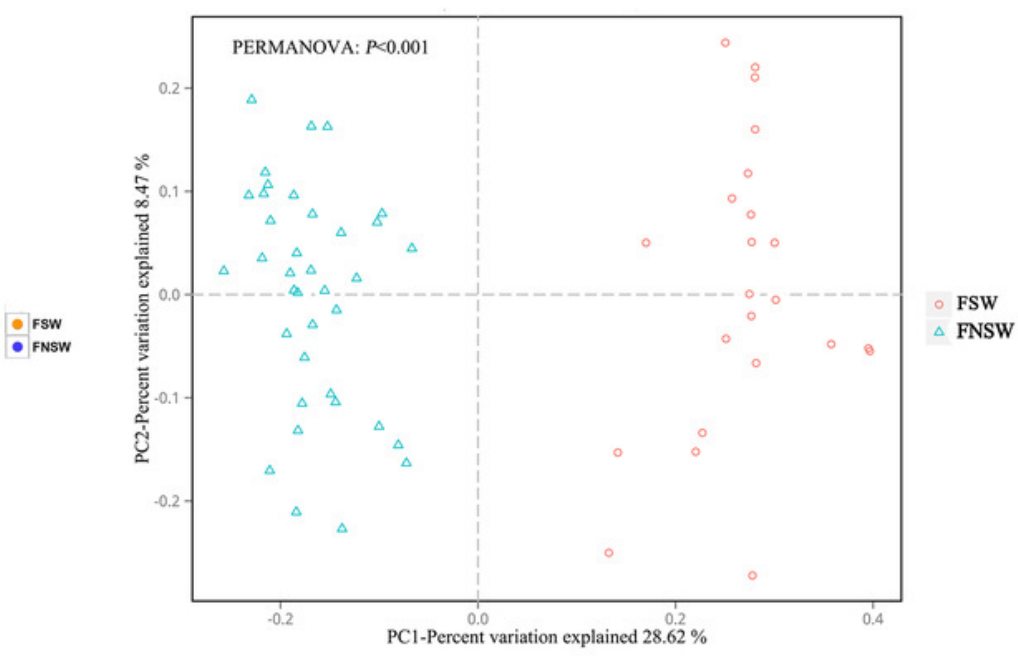

b )

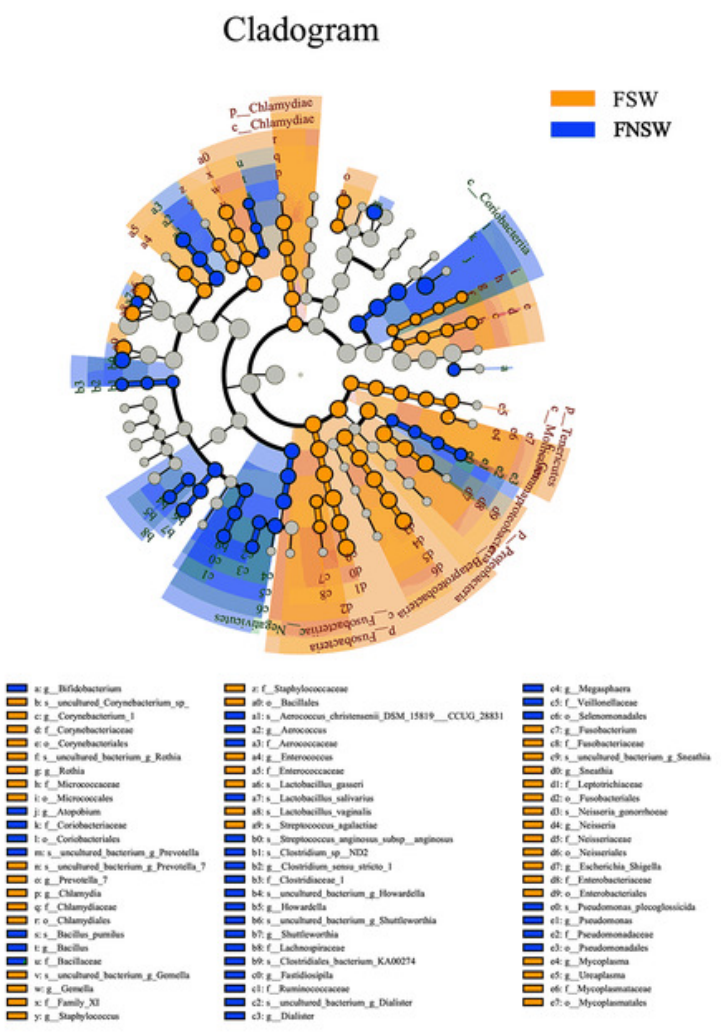

d) 
Figure 2

The specific genus between female sex workers and non-sex workers.

a) Lefse analysis for genus levels between FSWs and FNSWs, LDA score $>3.5 ;$ b)

MeanDecreaseGini index from random forest model; c) The average relative abundance of specific genera between FSWs and FNSWs; the asterisk relates to the P value of multivariate Kruskal-Wallis test was less than 0.05 by FDR adjustment. FSW, female sex worker; FNSW, non-sex worker.

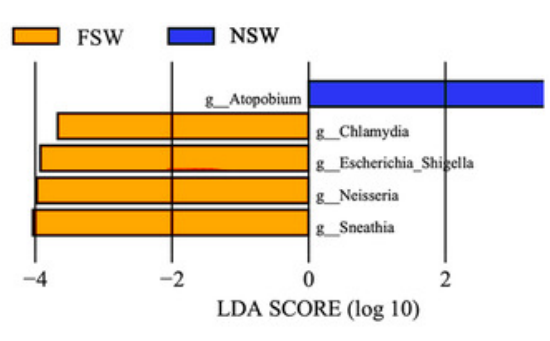

a )

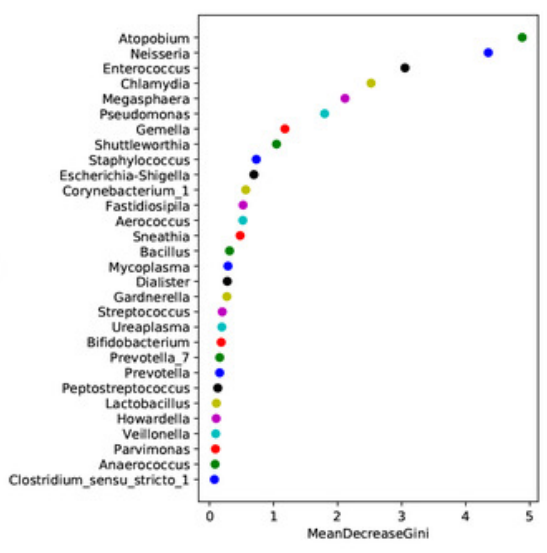

b )

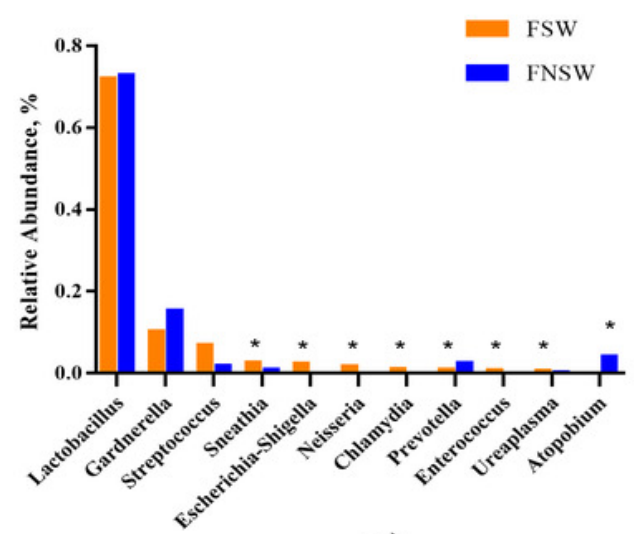

c ) 
Figure 3

The scatter diagrams of specific genera between female sex workers and non-sex workers.

The $P$ value was from multivariate Kruskal-Wallis test and adjusted by FDR. FSW, female sex worker; FNSW, female non-sex worker.

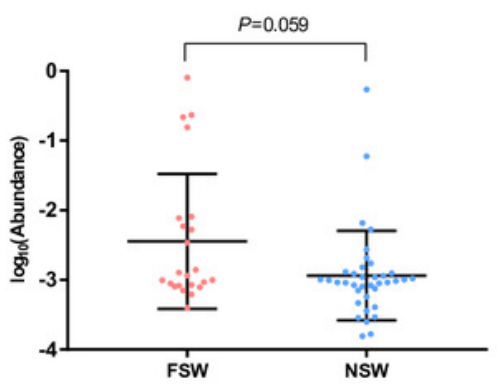

a) Streptococcus

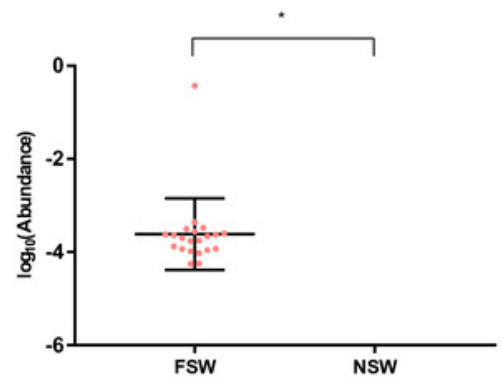

d) Neisseria

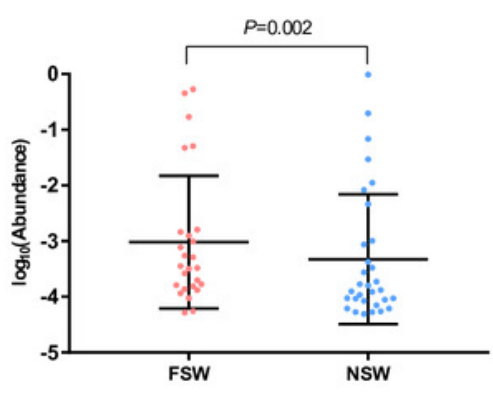

b) Sneathia

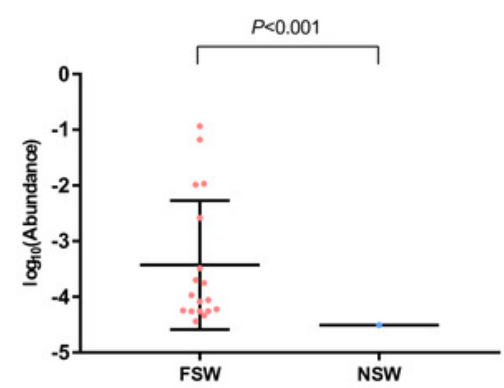

e) Chlamydia

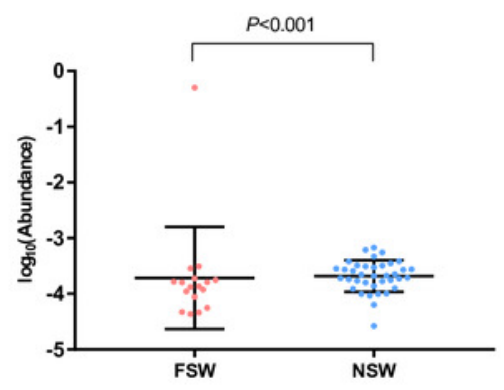

c) Escherichia-Shigella

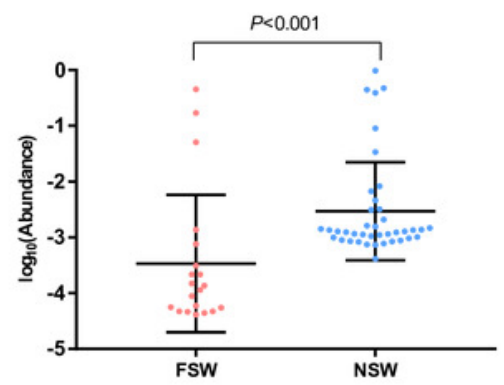

f) Atopobium 
Figure 4

The related abundance of Lactobacillus between female sex workers and non-sex workers.

The P value was from Kruskal-Wallis test. FSW, female sex worker; FNSW, female non-sex worker.

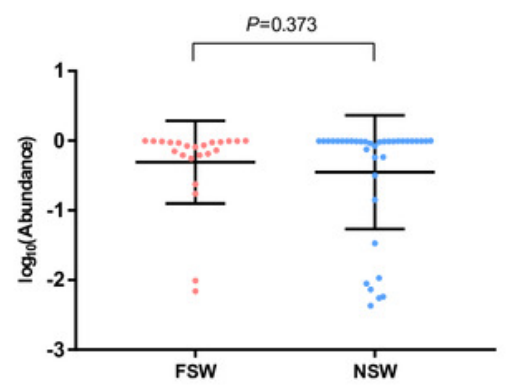

a) Lactobacillus

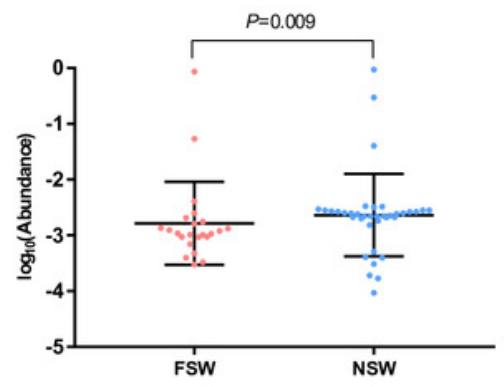

d) Lactobacillus gasseri

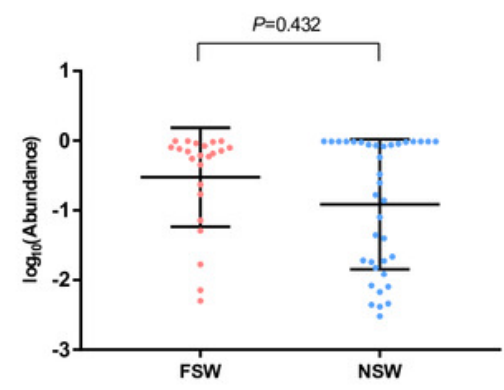

b) Lactobacillus iners

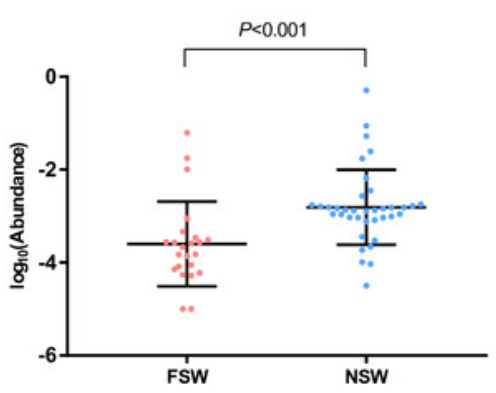

e) Lactobacillus jensenii

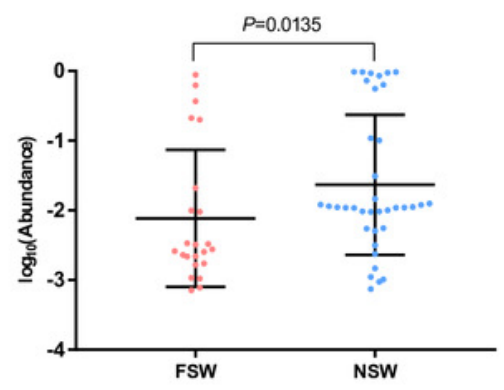

c) Lactobacillus crispatus

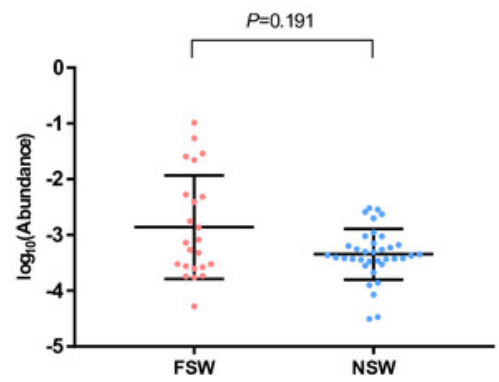

f) Other Lactobacillus 